\title{
The impact of government measures and human mobility trend on COVID-19 related deaths in the UK
}

\author{
Georgios M. Hadjidemetriou $^{\mathrm{a}, *, 1}$, Manu Sasidharan ${ }^{\mathrm{a}, *, 1}$, Georgia Kouyialis ${ }^{\mathrm{b}}$, Ajith K. Parlikad ${ }^{\mathrm{a}}$

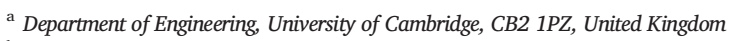 \\ b Schlumberger Cambridge Research, CB3 OEL, United Kingdom
}

\section{A R T I C L E I N F O}

\section{Article history:}

Received 7 May 2020

Received in revised form 29 June 2020

Accepted 29 June 2020

Available online 4 July 2020

\section{Keywords:}

COVID-19

Pandemic

Government restrictions

Travel behaviour

Logistic growth model

\begin{abstract}
A B S T R A C T
The COVID-19 global pandemic has rapidly expanded, with the UK being one of the countries with the highest number of cases and deaths in proportion to its population. Major clinical and human behavioural measures have been taken by the UK government to control the spread of the pandemic and to support the health system. It remains unclear how exactly human mobility restrictions have affected the virus spread in the UK. This research uses driving, walking and transit real-time data to investigate the impact of government control measures on human mobility reduction, as well as the connection between trends in human-mobility and severe COVID-19 outcomes. Human mobility was observed to gradually decrease as the government was announcing more measures and it stabilized at a scale of around $80 \%$ after a lockdown was imposed. The study shows that human-mobility reduction had a significant impact on reducing COVID-19-related deaths, thus providing crucial evidence in support of such government measures.
\end{abstract}

\section{Introduction}

On 31st December 2019, a group of pneumonia cases of unknown aetiology in Wuhan, Hubei Province, China was reported to the World Health Organization (WHO) China Office (W.H.O, 2020). By 7th January 2020, scientists in China had isolated a new coronavirus from patients in Wuhan (Wang et al., 2020). The virus is phylogenetically in the severe acute respiratory syndrome coronavirus (SARS-CoV) clade and the associated disease is stated as coronavirus disease 2019 (COVID-19). The rapid spread of the disease led the WHO to declare COVID-19 a global pandemic on 11th March 2020 (Eurosurveillance Editorial Team, 2020). As of 22nd June, more than 9 million cases of COVID-19 and more than 470,000 associated deaths were reported worldwide (European Centre for Disease Prevention and Control, 2020a). Given the high transmissibility and limited epidemiological understanding of COVID-19 (Arenas et al., 2020), governments across the world have adopted different social distancing measures to delay the transmission of the virus, including restrictions to human mobility and mass gatherings; closure of work- and entertainment-places; as well as cancellation and postponement of events. However, the effectiveness of such measures in controlling the pandemic is uncertain, with the decision-making surrounding the time and the extent of adoption being

\footnotetext{
* Corresponding author.

E-mail addresses: gh444@cam.ac.uk, (G.M. Hadjidemetriou),mp979@cam.ac.uk. (M. Sasidharan)

${ }^{1}$ Equal contribution.
}

rarely evidence-based (European Centre for Disease Prevention and Control, 2020b; Kraemer et al., 2020). The governments try to achieve a trade-off between the potential public health benefits of delaying COVID19 spread and the socio-economic impacts of social distancing measures (Sasidharan et al., 2020). These measures have resulted in a reduction of human mobility (De Vos, 2020; Klein et al., 2020; Warren and Skillman, 2020), arguably contributing to the delay in COVID-19 transmission (Chinazzi et al., 2020; Lai et al., 2020) and thereby to the decrease of pressure on health services (Queiroz et al., 2020).

The first case and the first associated death in the UK were reported on 31st January 2020 and 5th March 2020 respectively, with a total of more than 300,000 cases and 42,000 associated deaths being announced as of 22nd June (European Centre for Disease Prevention and Control, 2020a). With the impending risk of COVID-19 pandemic infecting up to $80 \%$ of the UK population (Banerjee et al., 2020), the government outlined its plans to support health and social care system through a COVID-19 Emergency Bill on 8th March 2020 (House of Commons, 2020). Fig. 1 shows the timescale of the main UK Government measures taken to tackle COVID-19 spread. The UK national framework for responding to pandemic states that public transport should continue to operate normally to maintain business continuity and near normal functioning of society as well as to enable critical workers' essential journeys (Department of Health and Social Care, 2020). The UK government's measures directly connected to human-mobility began on 16th March 2020, with a 'recommendation' on avoiding non-essential travel (HM Government, 2020). Additionally, the Transport for London (TfL) announced a reduced service across its transit 

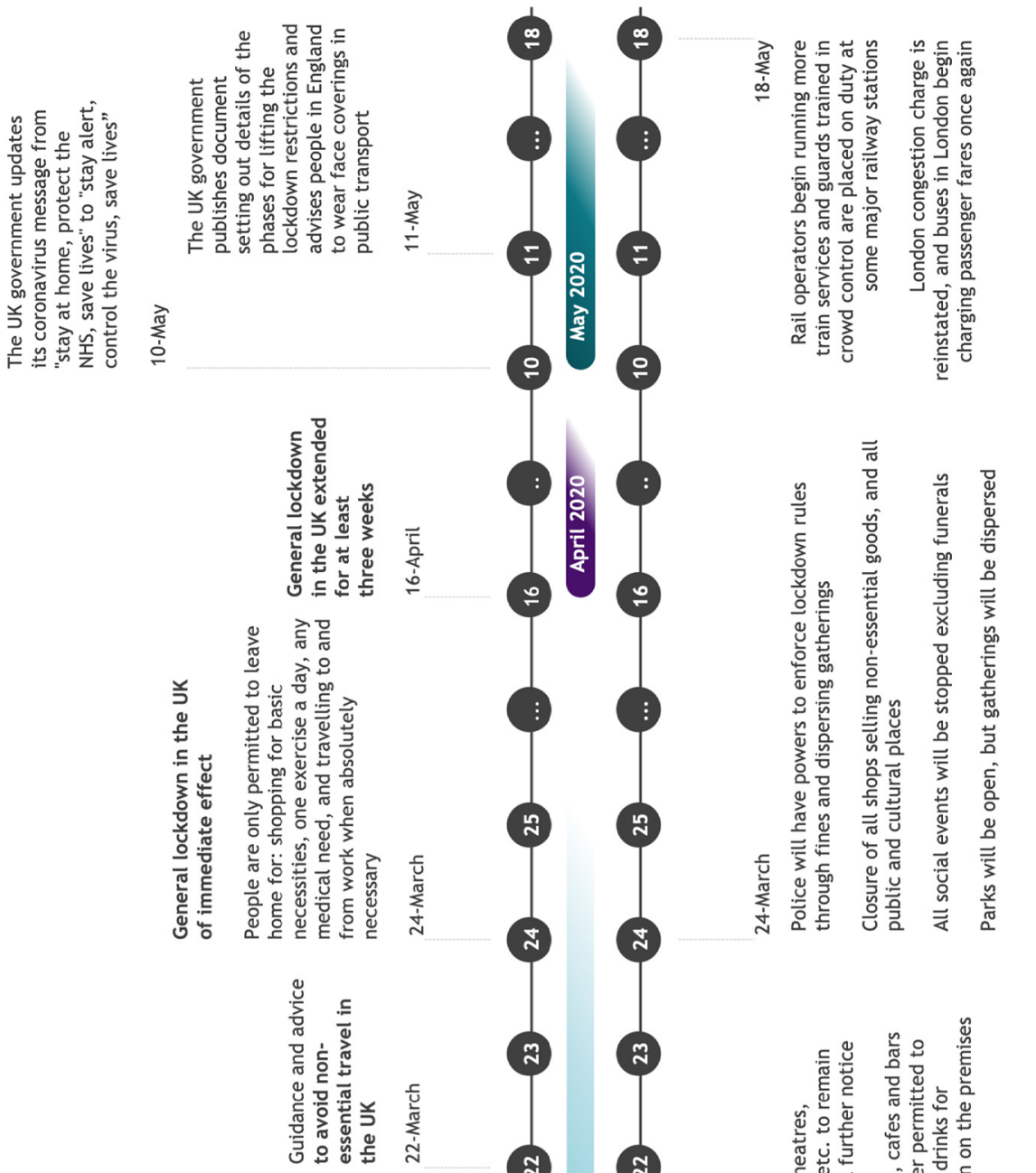

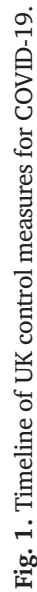
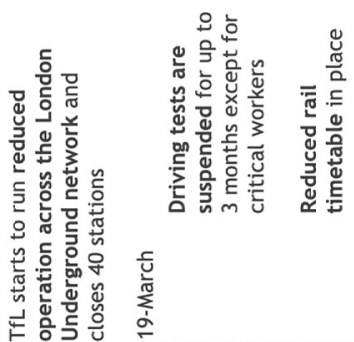

ส
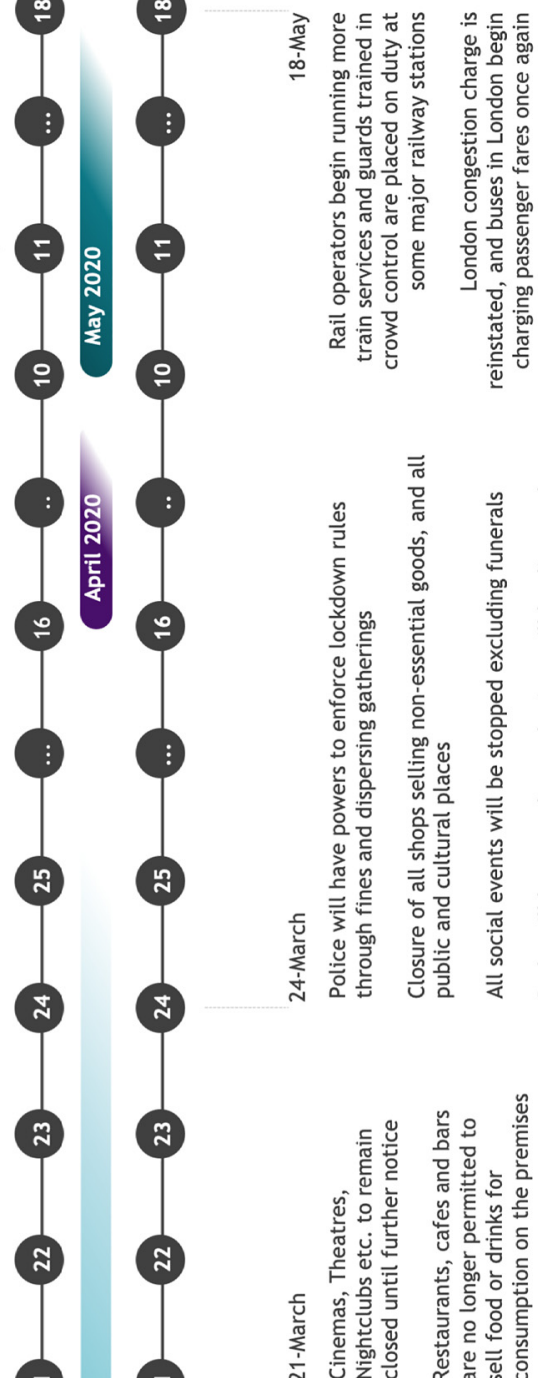

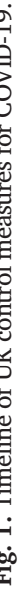

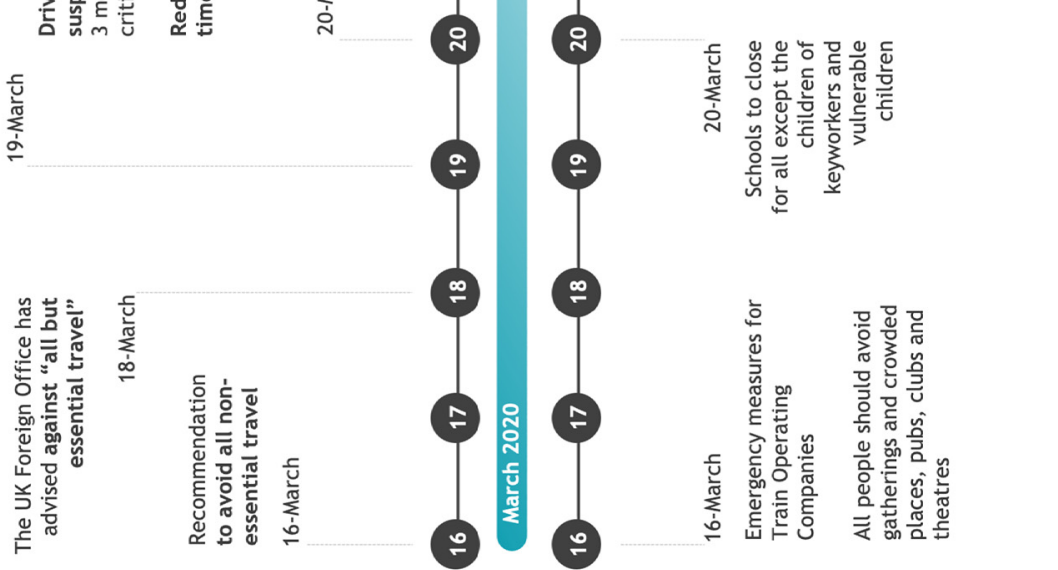
network on 18th March 2020 (Transport for London, 2020). It was followed by a reduced operation of the national railway network and cancellation of driving tests for up to three months. Subsequently, a general (nation-wide) lockdown was enforced with immediate effect on 23rd March 2020, accompanied by a 'guidance and advice' to avoid non-essential travel and to practice social distancing (European Commission, 2020). The lockdown was later extended on 16th April 2020 for at least three weeks. The study investigates the impact of the UK government's interventions on human mobility and its subsequent impact on the severe COVID-19 outcomes (i.e. deaths).

\section{Materials and methods}

The UK government's measures in response to COVID-19 was collected from publicly available databases (European Commission, 2020; HM Government, 2020) and illustrated in Fig. 1. The data associated with human mobility trends of walking, driving and using public transport provided by Apple mobility trends reports (Apple Maps, 2020) was compared to a baseline volume of the previous year (Fig. 2). The timeline of the government's measures was mapped against the human-mobility trends to study the plausible shifts in transport usage and mode choice.

The impact of the human-mobility trends on COVID-19 related deaths was investigated by employing reliable mathematical modelling, which captures the baseline transmission and predicts the effect of an epidemic (Pell et al., 2018; Viboud et al., 2016). The total period of an outbreak can be modelled with a logistic growth (Wu et al., 2020), characterized by increasing growth at the beginning and a decreasing one at a later stage. Generalized logistic model as an extension of the logistic model allows more flexibility to $\mathrm{S}$ shaped growth curves for asymmetrical growth (Szparaga and Kocira, 2018; Yin et al., 2003). The input parameters of the logistic function consist of the 7day moving average number of deaths at a given time, the limiting value of the number of deaths, the midpoint where the epidemic starts to slow down and the growth rate determining how many people each person infects. A nonlinear regression model is applied to the total number of 7-day moving average deaths in Period 1 (until 25th March) and Period 2 (until 10th April). Nonlinear least-squares estimation is used to generate the parameters that minimize the Least Squared Error of our model. Customized bounds and random initial points are deployed. The fitted functions with the predicted values over the entire period until 20th June and the full dataset are shown in Fig. 3. The optimization model and relevant functions were implemented in Python 3.7 using the Scipy library, and the source code is available in Hadjidemetriou et al. (2020).

\section{Results}

The results from our analysis indicate that most of the UK government measures were directly or indirectly connected with human mobility. Human mobility in the UK, particularly through public transport, was seen to be consistently higher in comparison to the previous year until the 7th March 2020, as illustrated in Fig. 2. During the same period, the severity of the pandemic in continental Europe was rising at an alarming rate, with Italy roughly doubling its COVID-19 related daily deaths every 2 to 3 days (European Centre for Disease Prevention and Control, 2020a). A notable drop in people driving and using public transport in the UK was observed from 8th March 2020. This observation may indicate that the government's press release on this date, outlining the COVID-19 Emergency Bill and plans to move from 'contain' to 'delay' the COVID-19 progression, had a direct impact on travel behaviour. The continuous UK government's 'guidance' and 'advice and recommendation' to avoid non-essential travel between 8th-23rd March 2020, combined with the closure of schools and reduced operation of London Underground and National Railway services, possibly contributed to a continuous reduction in human-mobility during this period. On 24th March 2020 (i.e. the day after the general lockdown), human mobility was found to be reduced even more and until the end of May 2020 did not show any major fluctuations, with driving, transit and walking remaining $60 \%, 80 \%$ and $60 \%$ reduced compared to the same period of the previous year (i.e. 2019).
Despite remaining unclear how exactly different non-pharmaceutical control measures, such as human-mobility restrictions, affect COVID-19 spread (Reno et al., 2020; Tian et al., 2020a), the interventions in China possibly played a significant role in its mitigation (Chinazzi et al., 2020; Kraemer et al., 2020). Given the above, we investigated whether there is a correlation between human-mobility and number of COVID-19 related deaths. It should be mentioned that the mean time between exposure to the COVID-19 virus and reported death is 18 days (Zhou et al., 2020). To this end, we examined the potential link between the human mobility data of a particular date and the 7-day moving average number of deaths reported 18 days later. The 7-day moving average number of deaths was selected over a daily number of deaths to reduce statistical error and inconsistency in reporting deaths (e.g. between weekend and weekdays). In addition, deaths were considered as a more reliable metric (Ku et al., 2020), and thus selected over reported daily infections, since the number of the actual infections is expected to be significantly higher than what has been reported. Most UK citizens with no or mild symptoms have not been tested, so it is not clear whether they have been symptomatic or asymptomatic COVID-19 carriers (Bai et al., 2020; Djalante et al., 2020), while in case of death the causes have been investigated and recorded. We hypothesised that travel behaviour influences the risk of exposure to the virus and thus correlates with COVID-19 related deaths. Specifically, reduced human mobility can be associated with a lower number of deaths, compared to the number of deaths if human mobility remains at its normal levels.

Fig. 3 firstly presents the actual increase of the daily deaths informed by the 7-day moving average for the period between 6th March 2020 and 20th June 2020. Secondly, Fig. 3 shows the prediction for the increase of deaths until 20th June, using the deaths data until 26th March, correlated with the traffic data until 8th March (Period 1). Thirdly, Fig. 3 indicates the prediction for the growth of deaths until 20th June, using the deaths data until 10th April, correlated with the traffic data until 23rd March (Period 2). The trend of deaths is continuously changing, while traffic is continually decreasing in Period 2 (Figs. 2B, 3). Thus, Period 2 could have been divided into more parts. However, the used Periods serve our purpose of proving that a decrease in travel mobility is related to a lower number of deaths 18 days later (i.e. 26th March and 10th April respectively). Specifically, if there was no human mobility reduction (Period 1), the current number of deaths would be several times higher than the current actual number of deaths. If human mobility continued to be at the levels of the period between 8th and 23rd March (i.e. before lockdown period, but with constant "recommendations for avoiding non-essential travelling") until today, the resulting number of deaths would be again higher than the current deaths number, but significantly lower compared to the number of deaths associated with normal mobility level. It is clear that the human mobility levels are linked to severe COVID-19 outcomes (i.e. death) (Figs. 2, 3). However, any estimation for the exact number of saved lives due to the already applied measures or hypothetical measures poses a high level of uncertainty. COVID-19 related deaths do not only depend on government measures and human mobility, but also population density, population age and preexisting health condition, provided treatment as well as health care facilities occupancy and accessibility (Docherty Annemarie et al., 2020; Murthy et al., 2020; Tian et al., 2020b).

\section{Concluding discussion}

The largest share of human-mobility reduction was observed between 8 and 23 March 2020, when the UK government was continuously updating its measures until the lockdown. While the number of COVID-19 related deaths is dependent on various factors, the results from this study show that initial control measures aimed at human-mobility reduction had a direct impact on the number of COVID-19 related deaths in the UK. However, the presented methodology can be generalized only after using a larger dataset, model validation and comparison with other performance metrics of different models. 
The results from the study also indicate that some level of travel restrictions and social-distancing measures may need to continue to reduce the risk of a resurgence in the transmission of COVID-19 in the UK. Restricting the number of passengers in public transport and stations to comply with social-distancing measures may need to be implemented to encourage public transport usage safely. Similarly, the increased opportunities for promoting active travel need to be met by cities re-allocating space to allow physically spaced walking and cycling. Further study is recommended on the estimation of saved lives in case of alternative measures (e.g. earlier lockdown) only if in addition to travel behaviour, other factors, such as population density, population age and pre-existing health condition, provided treatment as well as health care facilities occupancy and

100
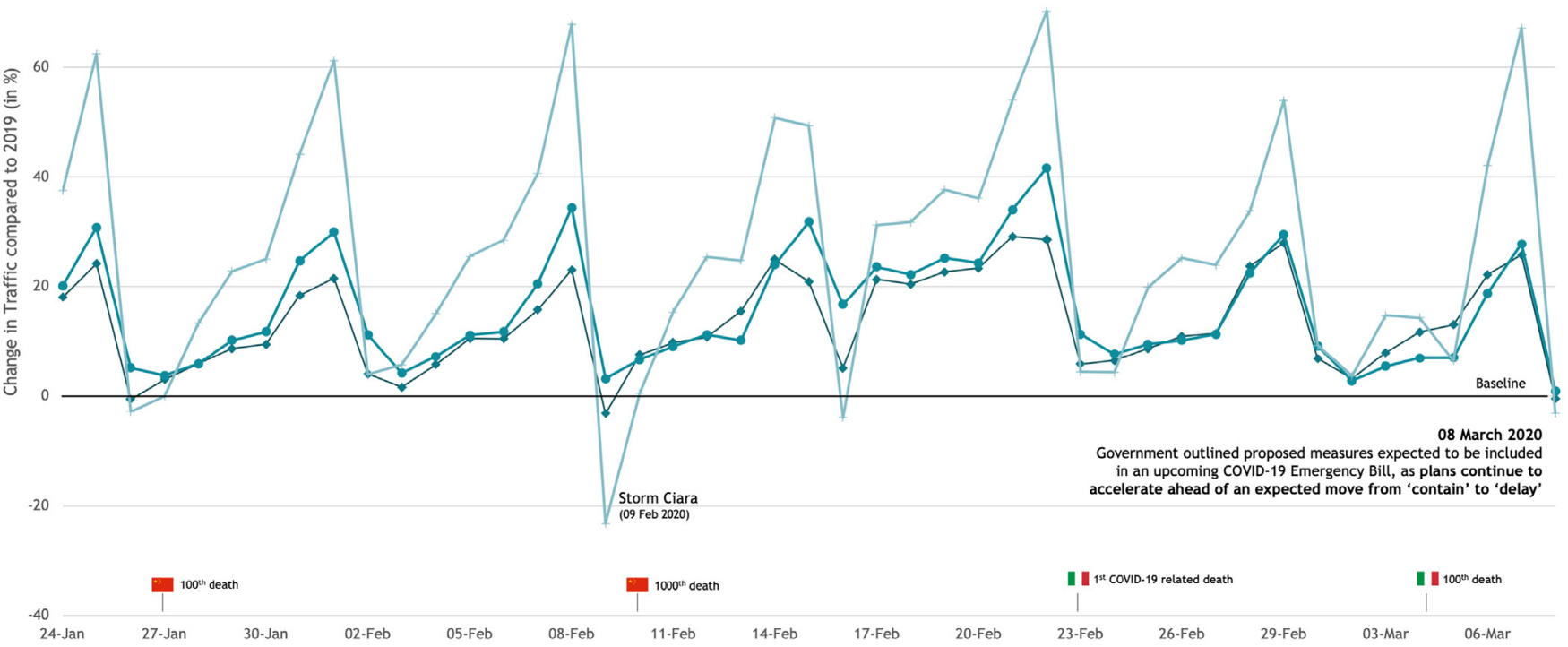

(A)

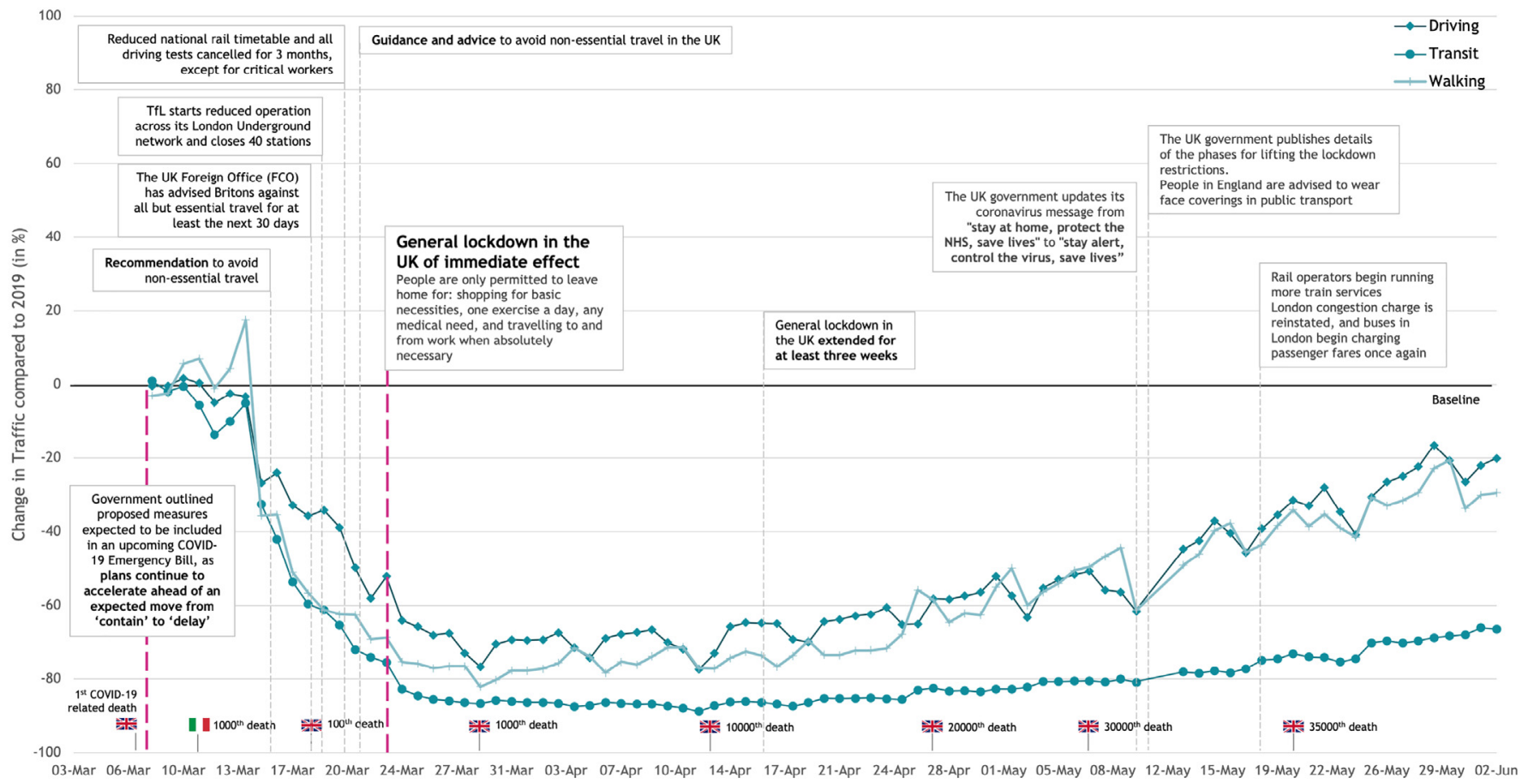

(B)

Fig. 2. Change in traffic in the UK compared to 2019: (A) before the 8th March 2020; (B) after 8th March 2020. 


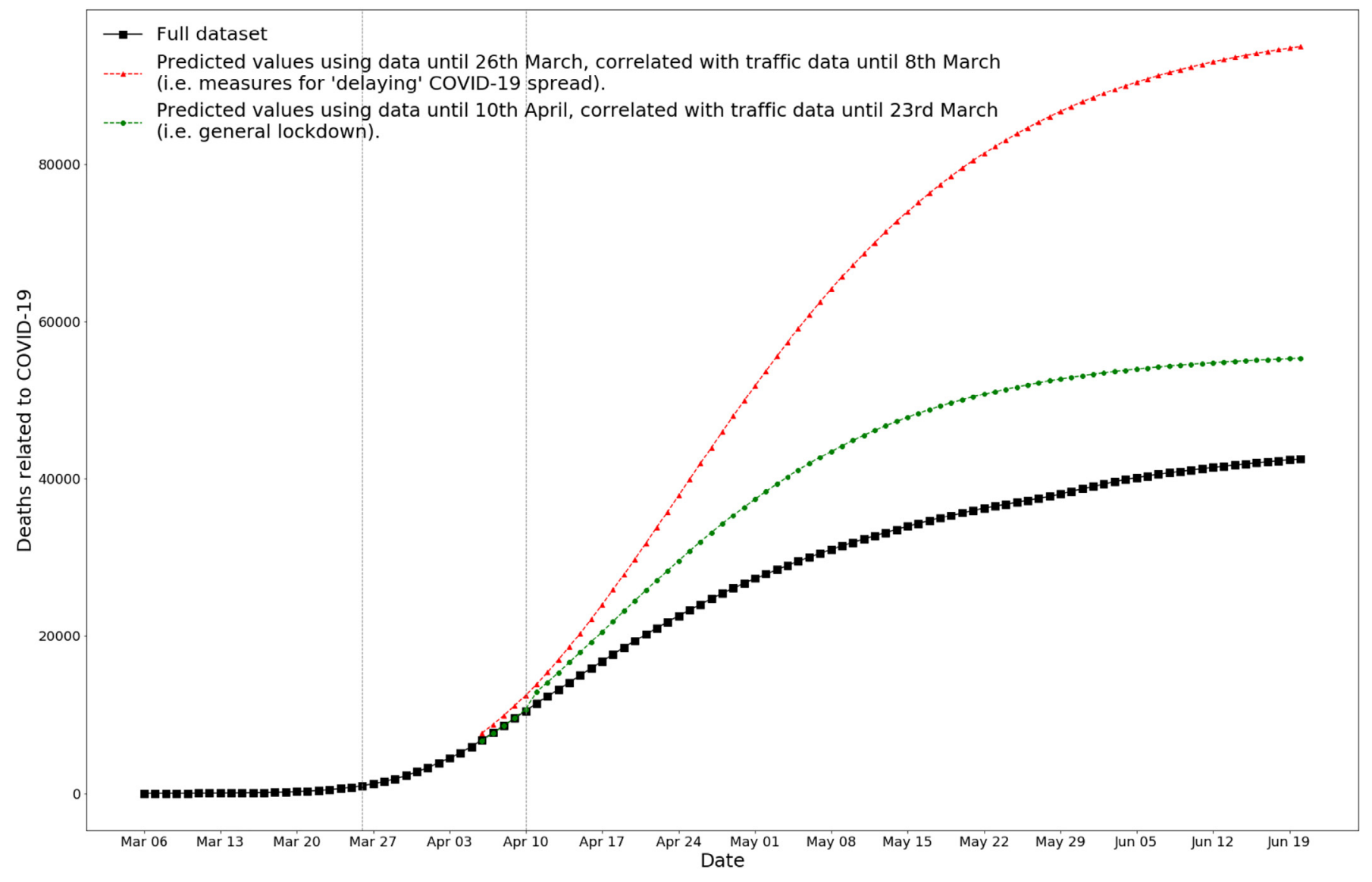

Fig. 3. Deaths related to COVID-19 in the UK: (A) actual values; (B) predicted values, using data until the 25th March; (C) predicted values, using data until the 10th April.

accessibility will be considered. Additionally, future work may investigate which individual measures or combination of them aid in achieving the trade-off between reducing human mobility and achieving near-normal functioning of socio-economic activities.

\section{Funding}

G. Hadjidemetriou's time on this research at the University of Cambridge was funded by the European Community's H2020 Programme MG7-1-2017 Resilience to extreme (natural and man-made) events [grant number 769255] - "GIS-based infrastructure management system for optimized response to extreme events of terrestrial transport networks (SAFEWAY)". M. Sasidharan's time on this research at the University of Cambridge was funded by the Engineering and Physical Science Research Council (EPSRC) through the grant EP/N021614/1 (CSIC Innovation and Knowledge Centre Phase 2) and Innovate UK through the grant 920035 (Centre for Smart Infrastructure and Construction).

\section{Data and materials availability}

The data collected for this analysis and the Python code are all publicly accessible in the links mentioned in the main text.

\section{CRediT authorship contribution statement}

Georgios M. Hadjidemetriou: Conceptualization, Methodology, Formal analysis, Data curation, Writing - original draft. Manu Sasidharan: Conceptualization, Methodology, Formal analysis, Data curation, Writing original draft. Georgia Kouyialis: Formal analysis, Data curation, Writing original draft. Ajith K. Parlikad: Conceptualization, Methodology.

\section{Declaration of competing interest}

Authors declare no competing interests.

\section{References}

Apple Maps, 2020. Mobility Trends Reports.

Arenas, A., Cota, W., Gomez-Gardenes, J., Gómez, S., Granell, C., Matamalas, J.T., SorianoPanos, D., Steinegger, B., 2020. A mathematical model for the spatiotemporal epidemic spreading of COVID19. medRxiv.

Bai, Y., Yao, L., Wei, T., Tian, F., Jin, D.-Y., Chen, L., Wang, M., 2020. Presumed asymptomatic carrier transmission of COVID-19. JAMA 323, 1406. https://doi.org/10.1001/ jama.2020.2565.

Banerjee, A., Pasea, L., Harris, S., Gonzalez-Izquierdo, A., Torralbo, A., Shallcross, L., Noursadeghi, M., Pillay, D., Sebire, N., Holmes, C., Pagel, C., 2020. Estimating excess 1year mortality associated with the COVID-19 pandemic according to underlying conditions and age: a population-based cohort study. Lancet https://doi.org/10.1016/S01406736(20)30854-0.

Chinazzi, M., Davis, J.T., Ajelli, M., Gioannini, C., Litvinova, M., Merler, S., y Piontti, A.P., Mu, K., Rossi, L., Sun, K., 2020. The effect of travel restrictions on the spread of the 2019 novel coronavirus (COVID-19) outbreak. Science 368 (6489), 395-400. https://doi. org/10.1001/jama.2020.2565.

De Vos, J., 2020. The effect of COVID-19 and subsequent social distancing on travel behavior. Transportation Research Interdisciplinary Perspectives 5, 100121. https://doi.org/ 10.1016/j.trip.2020.100121.

Department of Health and Social Care, 2020. Government outlines further plans to support health and social care system in fight against COVID-19.

Djalante, R., Lassa, J., Setiamarga, D., Sudjatma, A., Indrawan, M., Haryanto, B., Mahfud, C., Sinapoy, M.S., Djalante, S., Rafliana, I., Gunawan, L.A., Surtiari, G.A.K., Warsilah, H., 2020. Review and analysis of current responses to COVID-19 in Indonesia: period of January to March 2020. Progress in Disaster Science 6, 100091. https://doi.org/10.1016/j.pdisas.2020.100091.

Docherty Annemarie, B., Harrison Ewen, M., Green Christopher, A., Hardwick Hayley, E., Riinu, Pius, Lisa, Norman, et al., 2020. Features of 20133 UK patients in hospital with Covid-19 using the ISARIC WHO clinical characterisation protocol: prospective observational cohort study. BMJ 369, m1985.

European Centre for Disease Prevention and Control, 2020a. Data on the geographic distribution of COVID-19 cases worldwide.

European Centre for Disease Prevention and Control, 2020b. Considerations relating to social distancing measures in response to COVID-19 - second update. 
European Commission, 2020. Coronavirus Response: Travel and Transportation.

Eurosurveillance Editorial Team, 2020. Updated rapid risk assessment from ECDC on the novel coronavirus disease 2019 (COVID-19) pandemic: increased transmission in the EU/EEA and the UK. Eurosurveillance 25.

Hadjidemetriou, G.M., Sasidharan, M., Kouyialis, G., Parlikad, A.K., 2020. "Source code. available at. https://github.com/georgiakouy/COVID-19.

HM Government, 2020. Coronavirus (COVID-19): UK Government Response.

House of Commons, 2020. Coronavirus Bill - Explanatory Notes (BIll 122).

Klein, B., LaRocky, T., McCabey, S., Torresy, L., Privitera, F., Lake, B., Kraemer, M.U., Brownstein, J.S., Lazer, D., Eliassi-Rad, T., 2020. Assessing changes in commuting and individual mobility in major metropolitan areas in the United States during the COVID-19 outbreak.

Kraemer, M.U., Yang, C.-H., Gutierrez, B., Wu, C.-H., Klein, B., Pigott, D.M., du Plessis, L., Faria, N.R., Li, R., Hanage, W.P., 2020. The effect of human mobility and control measures on the COVID-19 epidemic in China. Science 368 (6490), 493-497.

Ku, C.C., Ng, T.-C., Lin, H.-H., 2020. Epidemiological benchmarks of the COVID-19 outbreak control in China after Wuhan's lockdown: a modelling study with an empirical approach. SSRN Journal. https://doi.org/10.2139/ssrn.3544127.

Lai, S., Ruktanonchai, N.W., Zhou, L., Prosper, O., Luo, W., Floyd, J.R., Wesolowski, A., Zhang, C., Du, X., Yu, H., 2020. Effect of non-pharmaceutical interventions for containing the COVID-19 outbreak: an observational and modelling study. medRxiv

Murthy, S., Gomersall, C.D., Fowler, R.A., 2020. 2020/care for critically ill patients with COVID-19. JAMA 323, 1499-1500. https://doi.org/10.1001/jama.2020.3633 JAMA.

Organization, W.H, 2020. Novel Coronavirus (2019-nCoV): Situation Report. 1.

Pell, B., Kuang, Y., Viboud, C., Chowell, G., 2018. Using phenomenological models for forecasting the 2015 Ebola challenge. Epidemics 22, 62-70.

Queiroz, L., Ferraz, A., Melo, J.L., Barboza, G., Urbanski, A.H., Nicolau, A., Oliva, S., Nakaya, H., 2020. Large-Scale Assessment of Human Mobility during COVID-19 Outbreak.

Reno, C., Lenzi, J., Navarra, A., Barelli, E., Gori, D., Lanza, A., Valentini, R., Tang, B., Fantini, M.P., 2020. Forecasting COVID-19-Associated Hospitalizations under Different Levels of Social Distancing in Lombardy and Emilia-Romagna. Results from an Extended SEIR Compartmental Model, Northern Italy.

Sasidharan, M., Singh, A., Torbaghan, M.E., Parlikad, A.K., 2020. A vulnerability-based approach to human-mobility reduction for countering COVID-19 transmission in London while considering local air quality. Sci. Total Environ. https://doi.org/10.1016/j. scitotenv.2020.140515.

Szparaga, A., Kocira, S., 2018. Generalized logistic functions in modelling emergence of Brassica napus L. PLoS One 13, e0201980.

Tian, H., Liu, Y., Li, Y., Wu, C.H., Chen, B., Kraemer, M.U.G., Li, B., Cai, J., Xu, B., Yang, Q., Wang, B., Yang, P., Cui, Y., Song, Y., Zheng, P., Wang, Q., Bjornstad, O.N., Yang, R., Grenfell, B.T., Pybus, O.G., Dye, C., 2020a. An investigation of transmission control measures during the first 50 days of the COVID-19 epidemic in China. Science, eabb6105 https://doi.org/10.1126/science.abb6105.

Tian, J., Yuan, X., Xiao, J., Zhong, Q., Yang, C., Liu, B., Cai, Y., Lu, Z., Wang, J., Wang, Y., Liu, S., 2020b. Clinical characteristics and risk factors associated with COVID-19 disease severity in patients with cancer in Wuhan, China: a multicentre, retrospective, cohort study. The Lancet Oncology. https://doi.org/10.1016/S1470-2045(20)30309-0.

Transport for London, 2020. London's Transport Commissioner Mike Brown on Our Response to Coronavirus.

Viboud, C., Simonsen, L., Chowell, G., 2016. A generalized-growth model to characterize the early ascending phase of infectious disease outbreaks. Epidemics 15, 27-37.

Wang, C., Horby, P.W., Hayden, F.G., Gao, G.F., 2020. A novel coronavirus outbreak of global health concern. Lancet 395, 470-473. https://doi.org/10.1016/S0140-6736 (20)30185-9.

Warren, M.S., Skillman, S.W., 2020. Mobility changes in response to COVID-19. arXiv preprint arXiv:2003.14228.

Wu, K., Darcet, D., Wang, Q., Sornette, D., 2020. Generalized logistic growth modeling of the COVID-19 outbreak in 29 provinces in China and in the rest of the world. arXiv preprint arXiv:2003.05681.

Yin, X., Goudriaan, J.A.N., Lantinga, E.A., Vos, J.A.N., Spiertz, H.J., 2003. A flexible sigmoid function of determinate growth. Ann. Bot. 91, 361-371.

Zhou, F., Yu, T., Du, R., Fan, G., Liu, Y., Liu, Z., Xiang, J., Wang, Y., Song, B., Gu, X., 2020. Clinical course and risk factors for mortality of adult inpatients with COVID-19 in Wuhan, China: a retrospective cohort study. Lancet 395, 1054-1062. 